\title{
Bridge Displacements Monitoring Using Space-Borne X-Band SAR Interferometry
}

\author{
Milan Lazecky, Ivana Hlavacova, Matus Bakon, Joaquim J. Sousa, Daniele Perissin, and Gloria Patricio
}

\begin{abstract}
The development of interferometric methodologies for deformation monitoring that are able to deal with long time series of synthetic aperture radar (SAR) images made the detection of seasonal effects possible by decomposing the differential SAR phase. In the case of monitoring of man-made structures, particularly bridges, the use of high-resolution X-band SAR data allows the determination of three major components with significant influence on the SAR phase: the linear deformation trend, the height of structures over terrain, and the thermal expansion. In the case of stable metallic or (reinforced) concrete structures, this last effect can reach a magnitude comparable to or even exceeding the other phase components. In this review, we present two case studies that confirm the feasibility of InSAR techniques for bridge deformation monitoring and our original approach to refine the thermal expansion component.
\end{abstract}

Index Terms-Displacement measurement, radar interferometry, remote sensing, road transportation, space-borne radar, synthetic aperture radar (SAR), thermal expansion.

\section{INTRODUCTION}

$\mathbf{I}$ $\mathrm{N}$ order to completely determine the condition of a bridge, a complex set of analyzes including various monitoring meth-

Manuscript received July 1, 2015; revised May 24, 2016; accepted June 23, 2016. Date of publication July 27, 2016; date of current version December 22,2016 . This work was supported in part by Operational Programme Education for Competitiveness, in part by the European Social Fund, in part by the state budget of the Czech Republic, in part by Disaster management support scenarios using geoinformation technologies No. VG20132015106 program promoted by the Ministry of Interior, Czech Republic, in part by European Regional Development Fund in the IT4Innovations Centre of Excellence project (CZ.1.05/1.1.00/02.0070), in part by European Fund for Regional Progress - FEDER (Fundo Europeu de Desenvolvimento Regional) through the project BI/COMPETE/38732/UTAD/2014 entitled "RemotWatch - Alert and Monitoring System for Physical Structures", in part by The Ministry of Education, Youth and Sports from the National Programme of Sustainability (NPU II) project "IT4Innovations excellence in science - LQ1602," in part by TB0100MD021 Beta and TB0400MD003 Beta from the Technology Agency of the Czech Republic, and in part by the Slovak Grant Agency VEGA under Projects 1/0714/15 and 1/0462/16. This paper has been elaborated in the framework of the projects "New creative teams in priorities of scientific research," reg. no.CZ.1.07/2.3.00/30.0055.

M. Lazecky is with the IT4Innovations, VSB-Technical University of Ostrava, 70833 Ostrava, Czech Republic (e-mail: milan.lazecky@ vsb.cz).

I. Hlavacova is with the Faculty of Civil Eng., CTU, Prague, 16629 Praha, Czech Republic and also with GISAT Ltd., 17000 Praha, Czech Republic (e-mail: hlavacova@insar.cz).

M. Bakon is with the Slovak University of Technology, 81243 Bratislava, Slovakia (e-mail: matus.bakon@ @stuba.sk).

J. J. Sousa is with the Universidade de Trás-os-Montes e Alto Douro, 5001801 Vila Real Portugal and INESC TEC (formerly INESC Porto), 4200 Porto, Portugal (e-mail: jjsousa@utad.pt).

D. Perissin is with the Lyles School of Civil Engineering, Purdue University, West Lafayette, IN 47907 USA (e-mail: perissin@ purdue.edu).

G. Patricio is with the Instituto Politécnico da Guarda, 6300-559 Guarda, Portugal and also with the, University of Porto, 4099-002 Porto, Portugal (e-mail: gpatricio@ipg.pt)

Color versions of one or more of the figures in this letter are available online at http://ieeexplore.ieee.org.

Digital Object Identifier 10.1109/JSTARS.2016.2587778 ods is necessary [1]. Space-borne synthetic aperture radar (SAR) interferometry (InSAR) allows a deformation analysis over areas spanning hundreds of kilometers with an accuracy of as high as $1 \mathrm{~mm} /$ year at low costs and retrospectively. It has the potential to play a key role in the development of effective bridge monitoring techniques or warning systems [2].

The main improvement achieved using the persistent scatterer interferometry (PSI) technique is that uniform deformations at low rates could be assessed more accurately [3]. However, for the difficulties in resolving high-phase gradients or non-uniform deformations [4], a new model for retrieving the deformation signal is needed. The influence of various seasonal sources of deformation, such as temperature changes, can be observed within PSI displacements time series [5]-[7]. In the case of metallic or reinforced concrete structures, it is known that the thermal expansion is often a significant component affecting the phase signature.

Up to now, several authors [8]-[12] have dealt with the thermal expansion of bridges or other structures monitored by InSAR-based techniques. The issue of separating the deformation and the thermal expansion is documented in InSAR analyzes of C-band data [13], [14]. Nevertheless, due to better spatio-temporal resolution and a shorter wavelength, current X-band SAR systems are more sensitive to thermal expansion [15].

Section II of this review deals with the most significant components affecting the interferometric phase in case of bridges, with a particular focus on the decomposition from line-of-sight (LOS) measurements and the thermal expansion component Section III-A introduces a novel technique of refining the component by finding the correlation between the InSAR phase residues and the estimated thermal expansion coefficient that can be found within an InSAR processing for some acquisition pairs and that indicates that the structure is expanding in an unexpected way. Section III-B continues in the topic by providing a method for either calibrating the temperature values or estimating a calibrated thermal expansion component that fits a physical model. Section IV presents an application of this methodology to two case studies where the thermal dilation component formed a major part of detected displacements:

\section{A. Radotín bridge, Prague (Czech Republic)}

Radotín bridge is a highway bridge located near the confluence of Moldau and Berounka rivers in Prague. It is built from steel and concrete, measuring over $2200 \mathrm{~m}$ (six segments), with a unique construction based on oblique pillars. Shortly, after putting into operation in September 2010, microscopic cracks 
in the welding of metal supports were found. For this case, 33 TerraSAR-X Staring SpotLight scenes were acquired with temporal resolution of 11 days within one year period (June 2014-June 2015).

\section{B. Svinov Bridges, Ostrava (Czech Republic)}

Deformations of Svinov bridges at the D1 highway roundabout in Ostrava occurred soon after its opening in 2008 and have been detected using PSI processing of Envisat dataset from 2008 to 2010 [5]. For follow-up work, TerraSAR-X Stripmap images since 2013 were ordered; current dataset includes $17 \mathrm{im}$ ages with mostly 33 days of temporal baseline within 1.5 year (October 2013-May 2015).

\section{INSAR PHASE COMPONENTS IN CASE OF BRIDGES}

In case of urban constructions, particularly bridges, monitored using high-resolution SAR data from current X-band missions, three major components influence SAR phase considerably: linear deformation trend, height difference (the residual height-induced phase difference after removal of the topography-related component) and the effect of thermal expansion. These components can be properly distinguished if: 1) appropriate number of SAR acquisitions is provided in the dataset; 2) none of the components exhibit extreme values; and 3 ) no significant correlation between the temporal, spatial, and temperature-based baseline vectors is present.

It is not easy to delineate borders between an experimental research and a reliable comprehension of these components that would quantify the ambiguous terms "appropriate number of acquisitions," "nonextreme values," and "no significant correlation." This needs to be considered by an InSAR operator on a case-to-case basis. To evaluate the applicability of InSAR for bridge monitoring, the operator should also work with terms such as the sensitivity of InSAR dataset for height estimation [16] or the accuracy of estimations based on the standard deviation of their residues [17] derivable from the temporal coherence [18].

The situation is even more complicated by taking into account the nonlinearity of bridge movements in time and space-within the first years after its construction, a bridge can settle down nonlinearly, with maximum movements in the middle of or on top of the bridge construction [19].

\section{A. Displacement From the Line-of-Sight Direction}

The effect of various sources of phase change differs based on the orientation toward the satellite LOS. The movements of a bridge are expected in 1) vertical direction, i.e., displacements due to construction subsidence, possible bending due to a temperature difference between the bottom and the top of a bridge, as well as potential bending due to significant differences in the load of the bridge; or in 2) horizontal direction, i.e., along the bridge direction, that is, displacements due to thermal expansion. Any detected variations from the expected displacements can be a sign of construction stability problems, especially in

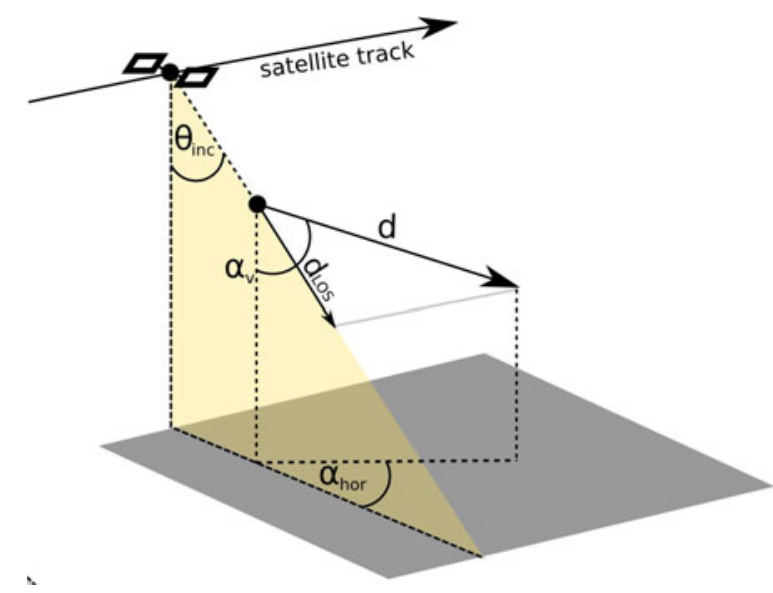

Fig. 1. Scheme of a movement detected in the SAR look direction $\left(d_{\mathrm{LOS}}\right)$, w.r.t., the direction of the real movement $(d)$.

case of horizontal movements in the direction perpendicular to the bridge.

In practice, high-resolution SAR acquisitions from both ascending and descending tracks, needed for a reliable 3-D decomposition [20], are often not available over the monitored area. A simplified conversion of displacement measurements from a single LOS direction, $d_{\mathrm{LOS}}$, into a displacement $d$ corresponding to the expected direction of the detected movement is performed as in the following equation, based on [21]:

$$
d=\frac{d_{\mathrm{LOS}}}{\cos \alpha_{v} \cos \theta_{\text {inc }}+\sin \alpha_{v} \cos \alpha_{\text {hor }} \sin \theta_{\text {inc }}}
$$

with $\theta_{\text {inc }}$ being the SAR incidence angle, $\alpha_{\text {hor }}$ the horizontal angle between the assumed orientation and the satellite LOS direction, and $\alpha_{v}$ the angle between the real movement and the vertical direction as depicted in Fig. 1.

The major thermal expansion is typically expected as a horizontal displacement component along the longitudinal axis of the bridge, $d_{h, \text { long }}$. The following equations obtain either $d_{h, \text { long }}$ or a rate of displacement in the vertical direction $d_{v}$ from $d_{\mathrm{LOS}}$, assuming no other movement affecting the $d_{\mathrm{LOS}}$ value at the time ( $\alpha_{v}$ is either 0 or $\left.\pi / 2 \mathrm{rad}\right)$ :

$$
\begin{aligned}
d_{h, \text { long }} & =\frac{d_{\mathrm{LOS}}}{\sin \theta_{\mathrm{inc}} \cdot \cos \alpha_{\mathrm{hor}}}=\frac{d_{\mathrm{LOS}}}{s_{h, \text { long }}} \\
d_{v} & =\frac{d_{\mathrm{LOS}}}{\cos \theta_{\mathrm{inc}}}=\frac{d_{\mathrm{LOS}}}{s_{v}} .
\end{aligned}
$$

To enhance the readiness of dependence of InSAR to the orientation of deformation vector, it is possible to establish the denominator of (1) as a sensitivity parameter, $s$, of $d_{\mathrm{LOS}}$ on real movement $d$. Then, the sensitivity $s_{h, \text { long }}$, the denominator of (2), describes the sensitivity of the movement of the bridge in the longitudinal direction to the satellite LOS, neglecting vertical movements, and similarly $s_{v}$, the denominator of (3), describes the sensitivity of InSAR in the vertical direction to the satellite LOS. 


\section{B. Introducing the Thermal Expansion Component}

Horizontal movements due to thermal expansion may cause significant difficulties in bridge displacement monitoring. Whereas the InSAR sensitivity is usually the highest for vertical movements, horizontal thermal expansion of bridges often exceeds centimeters, and in extreme cases even decimeters, making the thermal phase component hardly distinguishable from other sources of phase contribution, especially when the bridge direction is nearly parallel to the satellite LOS. In such cases, the thermal expansion appears as the major phase component and must be estimated in order to properly model other components such as the linear deformation trend, $k_{\text {defo }}$ [m/year], and the residual height estimation, $k_{\text {hei }}[\mathrm{m}]$.

Both $k_{\text {defo }}$ and $k_{\text {hei }}$ components are estimated in common multitemporal InSAR algorithms by finding a linear relation between (unwrapped) interferogram phase values $\Delta \Phi_{\mathrm{i}}[\mathrm{rad}]$ and either temporal differences $\Delta t_{i}$ [years] (in case of $k_{\text {defo }}$ ) or normal baselines $B_{i}[\mathrm{~m}]$ (for $k_{\mathrm{hei}}$ ) between each of the $i=1 \ldots N$ interferometric combinations of SAR images [22]. As implemented in the SARPROZ software [13], the approach for identifying the thermal expansion component, $k_{\text {temp }}\left[\mathrm{rad} /{ }^{\circ} \mathrm{C}\right]$, uses an additional parameter, searching also for a relation between $\Delta \Phi_{i}$ and differences of temperature values $\Delta T_{i}$ between the image acquisition times. These components are found as values maximizing the coherence as in (4), i.e., minimizing the phase residues, $\omega_{i}$, as in (5).

$$
\begin{aligned}
{\left[k_{\mathrm{defo}}, k_{\mathrm{hei}}, k_{\mathrm{temp}}\right]=} & \operatorname{argmax}\left|\frac{1}{N} \sum_{i=1}^{N} e^{j \omega_{i}}\right| \\
\omega_{i}= & \Delta \Phi_{i}+\frac{4 \pi}{\lambda}\left(k_{\mathrm{defo}} \Delta t_{i}-k_{\mathrm{hei}} \frac{B_{i}}{R \sin \theta_{\mathrm{inc}}}\right) \\
& -k_{\mathrm{temp}} \Delta T_{i}
\end{aligned}
$$

where $\lambda$ is the base wavelength of the SAR system and $R$ is the coarse minimal distance between the point and the SAR sensor.

\section{THERMAL EXPANSION COMPONENT REFINEMENT}

Based on experience acquired from the application of the PSI technique to bridge displacement monitoring, we propose an approach to decrease phase residues by refining the temperatures (A), and to calibrate the temperature differences in order for the estimated $k_{\text {temp }}$ value to conform to physical properties of the bridge (B).

\section{A. Temperature Refinement}

The thermal expansion is estimated as $k_{\text {temp }}$ on the basis of the measured temperature, assuming a linear relation between the temperature and the SAR phase. However, temperature values of a bridge construction are rarely available, and in addition, the temperature may differ in each part of the construction.

In our case studies, both air and surface temperature was measured at weather stations installed directly on bridge A and at a road in nearby surroundings of bridge $\mathrm{B}$, and are reported at the acquisition times with a 5-min tolerance. Differences of up to $11^{\circ} \mathrm{C}$ between the air temperature $T_{\text {air }}$ and the road surface

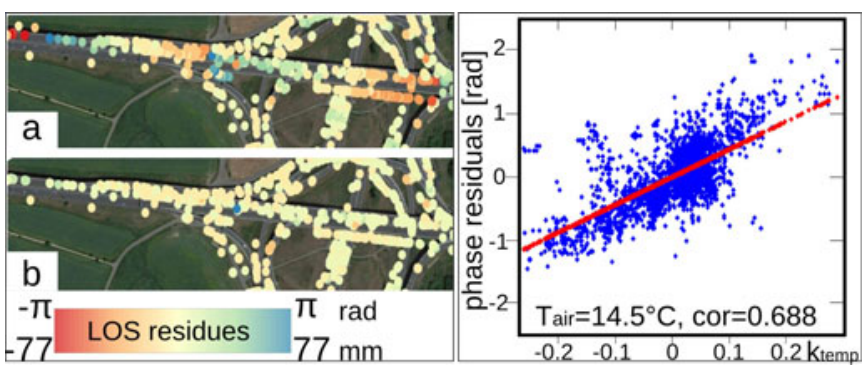

Fig. 2. Residues of November 09, 2014-May 26, 2015 interferogram over Radotin bridge after removal of estimated $k_{\mathrm{hei}}, k_{\mathrm{defo}}$, and $k_{\text {tem }}$ parameters using air temperature $\left(T_{\mathrm{air}}\right)$ values (a) before and (b) after the temperature refinement; right subfigure: coarse correlation between phase residues with estimated $k_{\text {temp }}$ using original $T_{\text {air }}$.

temperature $T_{\text {sur }}$ have been observed. Root mean square (RMS) of phase residues $\omega$ (i.e., phase values after removal of $k_{\mathrm{temp}}$, $k_{\text {defo }}$, and $k_{\text {hei }}$ estimates) was in both study sites lower when the $T_{\text {sur }}$ temperature values were used.

However, in both $T_{\text {air }}$ and $T_{\text {sur }}$ cases, significant spatial patterns similar to the pattern of $k_{\text {temp }}$ coefficients were observed in individual spatial plots of phase residues for some interferograms (an example is shown in Fig. 2). These can be attributed to nonmodeled displacements: such a pattern may be an indication of a nonlinear thermal expansion [9], but it can also be caused by an inaccurate temperature value, which is attributed to the difference between the air or road surface temperature and the temperature of the bridge structure itself. The sole fact that phase residues are correlated with some parameter is a sign of an inappropriate model.

In [25], a possibility of a temperature refinement according to the data is mentioned; however no particular model is presented. In case of wrong temperature values, residual phase values of PS points for a selected interferogram grow in relation with their estimated $k_{\text {temp }}$. The temperature values can be corrected through finding a correlation between phase residues $\omega$ of an interferogram and $k_{\text {temp }}$ of the PS points (selected based on the temporal coherence threshold). Their relation can be visualized for each interferogram $i$ as a cloud of points as shown on the right part of Fig. 2. The dependence of both values can be approximated by a line as follows:

$$
\omega_{i}=a_{i} \cdot k_{\mathrm{temp}}+b_{i}
$$

with $a_{i}$ and $b_{\mathrm{i}}$ being the least-squares regression results- $a_{i}$ can be directly applied as the temperature correction parameter for the $i$ th acquisition pair. In the presented test areas, the convergence was achieved in two iterations, for both temperature sets. The achieved refined temperatures differ if based on $T_{\text {air }}$ or on $T_{\text {sur }}$. The reestimated $k_{\text {temp,ref }}$ values differ as well for both sets. However, the phase residues are almost the same (and minimized) in both cases. After the refinement step, the RMS of the phase residues decreased by $5-60 \%$ and the coherence increased for most of points, as seen in Fig. 3 (the most significant increase was observed for $T_{\text {air }}$; for that reason, only the refined $T_{\text {air,ref }}$ is presented).

It was found out that a ratio $p$ between the two values of $k_{\text {temp }}$ estimated using both refined air $\left(k_{\text {temp,air,ref }}\right)$ and surface 

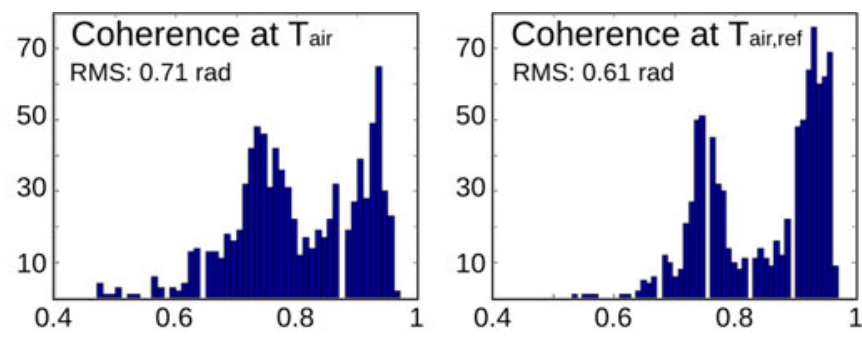

(a)
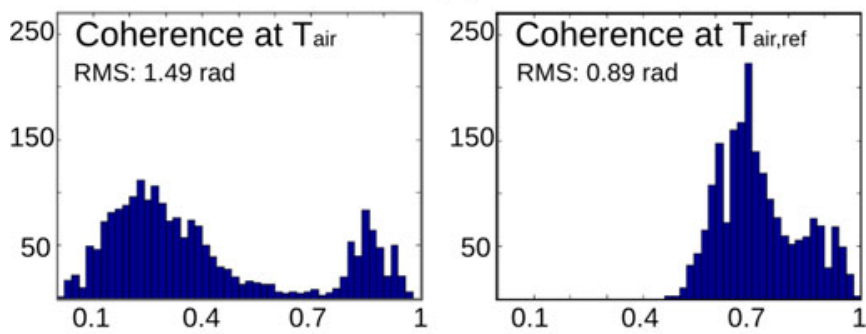

(b)

Fig. 3. Coherence histograms of PS for sites A and B before and after the refinement of air temperature data.

$\left(k_{\mathrm{temp}, \mathrm{sur}, \mathrm{ref}}\right)$ temperature sets $\left(T_{\mathrm{air}, \mathrm{ref}}, T_{\mathrm{sur}, \mathrm{ref}}\right)$ conforms to the relation (7). The $p$ ratio is approximately the same for $k_{\text {temp }}$ estimations of any PS point and approximately the same for $\Delta T$ of any interferogram as

$$
p=\frac{k_{\text {temp, air,ref }}}{k_{\text {temp,sur,ref }}}=\frac{\Delta T_{\text {sur,ref }}}{\Delta T_{\text {air,ref }}}
$$

\section{B. Calibration Based on Thermal Expansion Model}

To make a simple model of thermal expansion in the horizontal plane, it is possible to use the coordinates of the expansion joints, and then, compute the position of expansion centers, assumed to be in between the joints. The bridge segment between the expansion joints should behave based on a thermal expansion model, $t_{1}$, as

$$
t_{l}=c \cdot l \cdot \Delta T
$$

where $c$ is the thermal expansion coefficient for the material (e.g., $c=12 \times 10^{-6} \mathrm{~K}^{-1}$ for steel/concrete), $l$ is the length of the expanding structure, and $\Delta T$ is the structure temperature change causing the expansion.

Afterwards, the phase component of the thermal expansion model $k_{\text {temp,mod }}$ (9) for a selected PS point in the longitudinal distance $l$ from the expansion center is

$$
k_{\mathrm{temp}, \bmod }=c \cdot l \cdot s_{h, \text { long }} \cdot \frac{4 \pi}{\lambda} .
$$

In special cases of small or short data series, e.g., less than 1 year, the $k_{\text {temp,mod }}$ may substitute $k_{\text {temp }}$ estimations. In such cases, however, precise structure temperature measurements and a proper coefficient $c$ are necessary.

The comparison of the $k_{\text {temp,mod }}$ model and $k_{\text {temp }}$ based on the refined $T_{\text {air }}$ data for sites A and B is shown in Fig. 4. In the case of site $\mathrm{A}$, a feature of two correlation alignments (lines) appeared. In the spatial analysis, it was found out that

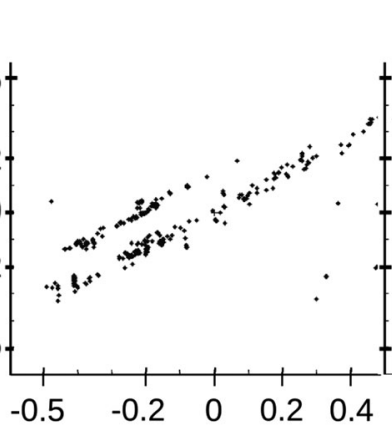

(a)

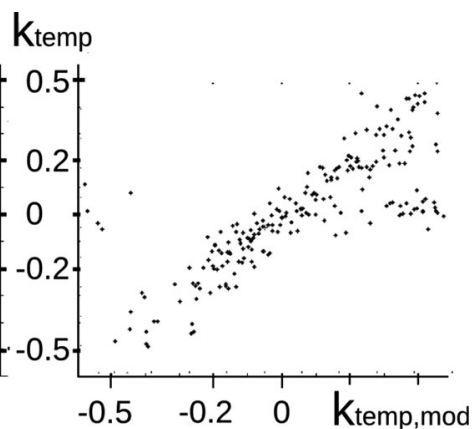

(b)
Fig. 4. Thermal expansion model-based coefficient $k_{\mathrm{tem}, \bmod }(x$-axis), w.r.t., InSAR-based coefficient $k_{\text {temp }}$ ( $y$-axis) for PS points of coherence $>0.8$ of sites $\mathrm{A}$ and $\mathrm{B}$, using values of refined air temperatures.
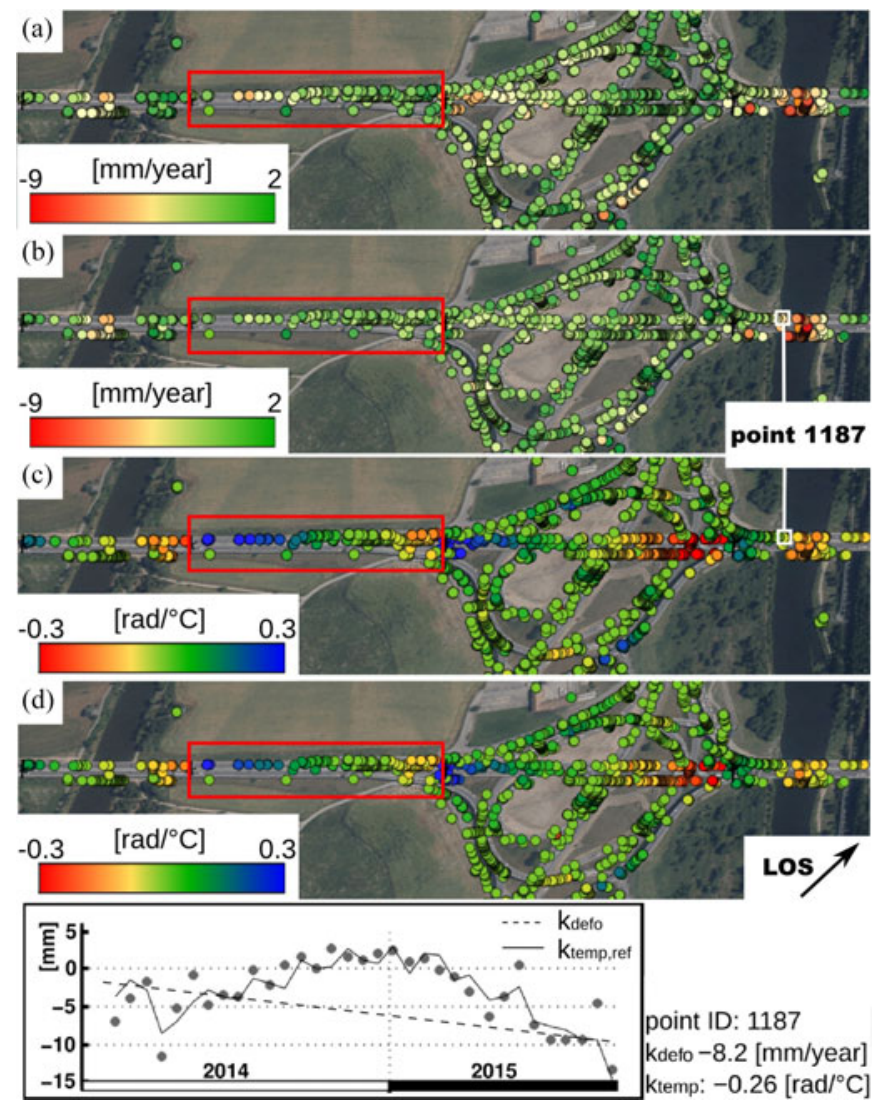

Fig. 5. From top to bottom: Linear deformation trend $k_{\text {defo }}$ computed (a) before and (b) after temperature refinement; (c) thermal expansion component based on original temperature values $k_{\text {temp,air }}$; and (d) refined $k_{\text {tem } \text {, air,ref }}$ for points of coherence $>0.7$ of the Radotín bridge. Below are the measured time series of the selected point 1187 over a river including both $k_{\text {defo }}$ and $k_{\text {temp,ref }}$ models and suggesting the major cause of estimated movement to be due to seasonal changes. The red rectangle marks the segment with a detected shift of the thermal expansion center (the slight shift is not visible here).

the shorter (not centered) line corresponds to a bridge segment that is expanding eccentrically. The cause of this effect is not known. The distance between the center of the segment and its thermal expansion center was estimated to be approximately $80 \mathrm{~m}$. The affected points are marked by a red rectangle in Fig. 5. 
Using $k_{\text {temp.mod }}$ in (7), it is possible to recalculate (calibrate) the preferably refined $T_{\text {ref }}$ values used to estimate refined parameter $k_{\text {temp,ref }}$ according to

$$
\Delta T_{\text {cal }}=\frac{k_{\text {temp,ref }}}{k_{\text {temp }, \text { mod }}} \cdot \Delta T_{\text {ref }}
$$

theoretically, the calibrated temperature values $T_{\text {cal }}$ should give the real values of the structure temperature, assuming accurate $c$ (8), and their use in (4) and (5) leads to the calibrated parameter $k_{\text {temp,cal }}$.

\section{Application to CASE Studies}

The progress leading to the presented case study results has been documented already in [2], [5], [23], and [25]. For both case studies, the refinement of $T_{\text {air }}$ has been applied based on investigation of $\omega$ by (6) after estimation of $k_{\mathrm{temp}}$ and $k_{\mathrm{hei}}$ and $k_{\text {defo }}$ parameters using (5). Because a one-year cycle (case of site $\mathrm{A}$ ) is not sufficient to distinguish between $k_{\text {defo }}$ and $k_{\text {temp }}$ (the separation is unstable), the inappropriately estimated $k_{\text {defo }}$ has been neglected from the refinement process at the site A.

Calibration results are not presented since these only change the absolute values of $k_{\mathrm{temp}}$.

\section{A. Radotín Bridge, Prague (Czech Republic)}

LOS sensitivity to horizontal movements in the direction along the bridge was computed as $s_{h, \text { long }}=49 \%$ (2), for vertical movements $s_{v^{\sim}}=85 \%$ (3). Previous PSI processing of 8-months TerraSAR-X data showed a high correlation between $k_{\text {defo }}$ and $k_{\text {temp }}$ [23]. In the current one-year dataset, parameters $k_{\text {defo }}$ and $k_{\text {temp }}$ still correlate [25].

Fig. 5 shows the results before and after the temperature refinement. After the refinement, the final $k_{\text {defo }}$ does not contain the spatial pattern between expansion joints. For short segments crossing the rivers, significant $k_{\text {defo }}$ as well as significant difference between $k_{\mathrm{temp}}$ and $k_{\mathrm{temp} \text {,mod }}$ was detected. The separation of $k_{\text {defo }}$ and $k_{\text {temp }}$ is not stable for a 1-year data range and in such a case, accurate temperature measurements are required to correctly separate these two components. Probably, due to the presence of water, the construction temperature is different for the segments above rivers; however, the computation assumes one temperature for the whole area.

\section{B. Svinov Bridges, Ostrava (Czech Republic)}

LOS sensitivity for the direction along the bridge ID D1-433 (bridge B) has been computed as $s_{h, \text { long }}=40 \%$ (2). Previous PSI processing of first 13 images (1-year cycle) showed issues separating $k_{\text {defo }}$ and $k_{\text {temp }}$ [23].

Fig. 6 presents $k_{\text {temp }}$ and $k_{\text {defo }}$ of points with coherence $>0.8$. The refinement increased the coherence of points (see Fig. 3). Values of $k_{\text {defo }}$ remain similar before and after the refinement. Thermal expansion joints can be clearly detected at the endpoints of $k_{\text {temp }}$ ramps.

Linear deformation trend of point ID 3282 is probably due to reflections from a metallic handrail at the slope of the bridge ramp. The slope leans on the bridge ramp wall. A wide crack
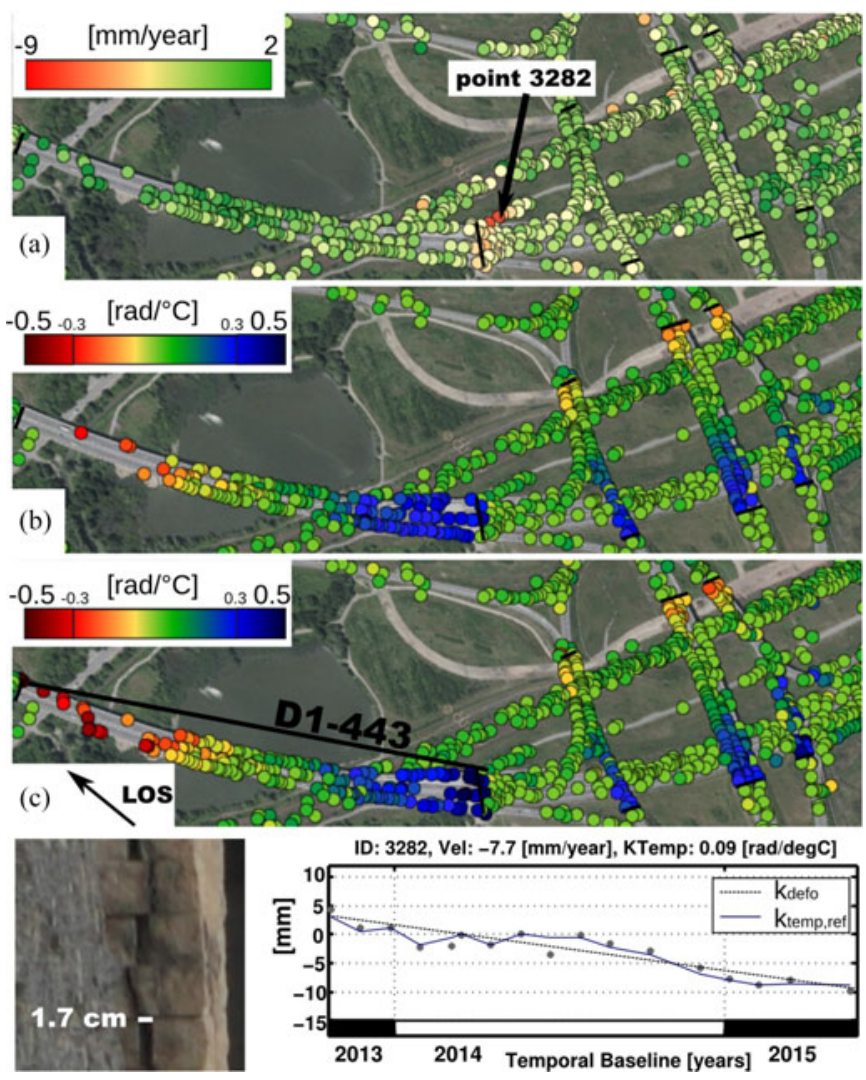

Fig. 6. From top to bottom: (a) linear deformation trend $k_{\text {defo }}$ (computed after temperature refinement); (b) thermal expansion component based on original

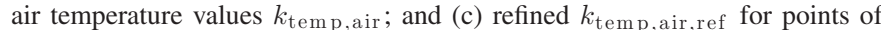
coherence $>0.8$ of Ostrava-Svinov bridges (increase of coherent points after refinement is visible at bridge D1-443). Below are the measured time series of point ID 3282 (at the bridge ramp wall affected by cracks).

in the wall has been spotted during a site visit in June 2015, as presented in Fig. 6. Points around the nearby joint move in $k_{\mathrm{defo}} \approx-5 \mathrm{~mm} /$ year.

\section{DISCUSSION}

We have neglected the variable delay due to atmosphere (APS) in our work in order to prevent the removal of thermal expansion part that would be modeled into APS (both contributions share a similar character of a spatial correlation without a temporal trend). It is known that APS varies slowly in space [24] but indeed it can affect the temperature refinement results even in the local scale. In order to increase the reliability of $k_{\mathrm{temp}, \mathrm{ref}}$, we recommend to estimate APS especially in case of monitoring of very long bridges, yet we do not recommend using points attached to an expanding bridge for the APS estimation. It can be neglected in case of shorter bridges - we have not detected any significant loss of coherence at distances lower than $500 \mathrm{~m}$ from the reference point.

The temperature refinement proposed here would work for structures represented by a sufficient number of points with a significant rate of the thermal expansion. These structures should expand linearly with temperature. Displacements of corresponding points should not be of a similar spatial pattern as the thermal expansion. 
We assume that at least a 1.5-year cycle is necessary to achieve a reliable estimation of (independent) InSAR parameters for bridges. In shorter periods, estimated $k_{\text {defo }}$ and $k_{\text {temp }}$ overlap. As observed in case study A, the temperature refinement significantly reduces this overlap.

\section{CONCLUSION}

Thermal expansion is a significant component in case of bridges monitored by X-band InSAR. Estimated as $k_{\text {temp }}$, its reliability depends on correct temperature values $T$. We propose a novel method to refine and calibrate $T$, and thus, $k_{\text {temp }}$ itself. The refinement of $k_{\text {temp }}$ improves the coherence of points and helps to accurately evaluate the linear deformation of a monitored bridge.

Similarly to [26], our method does not take into account the variance of temperature within the monitored structure. Assuming temperature homogeneity, its refined values are corrected relatively, irrespective of temperature input data, and differ only in their absolute values. The $k_{\text {temp, mod }}$ thermal expansion model can find its use in case of a limited number or time range of SAR images. Its reliability depends on appropriate structure temperature values (can be based on the refinement outputs) and a proper thermal expansion coefficient. Once reliable, it can be used to calibrate the InSAR-based $k_{\text {temp }}$ or even detect deformations in a linear trend with temperature.

\section{ACKNOWLEDGMENT}

TerraSAR-X data for this work were provided by the Deutsches Zentrum für Luft- und Raumfahrt (projects LAN2379, LAN1130). Data have been processed by SARPROZ (Copyright (c) 2009 Daniele Perissin, www.sarproz.com).

\section{REFERENCES}

[1] T. M. Ahlborn et al., "An evaluation of commercially available remote sensors for assessing highway bridge condition," Dept. Civil Environ. Eng., Michigan Tech. Univ., Houghton, MI, USA, Tech. Rep. DTOS5910-H-00001, Oct. 2010.

[2] J. J. Sousa et al., "Potential of multi-temporal InSAR techniques for bridges and dams monitoring," in Proc. Conf. Enterprise Inform. Syst., Troia, Portugal, 2014, pp. 834-831.

[3] U. Wegmuller, D. Walter, V. Spreckels, and C. Werner, "Nonuniform ground motion monitoring with TerraSAR-X persistent scatterer interferometry," IEEE Trans. Geosci. Remote Sens., vol. 48, no. 2, pp. 895-904, Feb. 2010.

[4] V. Spreckels, J. Musiedlak, U. Wegmuller, T. Strozzi, and C. Wichlacz, "Detection of underground coal mining-induced surface deformation by differential InSAR data," presented at the ISPRS WG I/2,I/5,IV/7 Workshop on High Resolution Mapping From Space, Hannover, Germany, Sep. 2001.

[5] M. Lazecky, P. Rapant, D. Perissin, and M. Bakon, "Deformations of highway over undermined Ostrava-Svinov area monitored by InSAR using limited set of SAR images," in Proc. Conf. Enterprise Inform. Syst., Troia, Portugal, 2014, pp. 414-421.

[6] M. Bakon, D. Perissin, M. Lazecky, and J. Papco, "Infrastructure nonlinear deformation monitoring via satellite radar interferometry," in Proc. Conf. Enterprise Inform. Syst., Troia, Portugal, 2014, pp. 294-300.

[7] D. Notti, C. Meisina, F. Zucca, M. Crosetto, and O. Montserrat, "Factors that have an influence on time series," presented at the Fringe, Frascati, Italy, Sep. 2011.
[8] D. Reale, G. Fornaro, and A. Pauciullo, "Extension of 4-D SAR imaging to the monitoring of thermally dilating scatterers," IEEE Trans. Geosci. Remote Sens., vol. 51, no. 12, pp. 5296-306, Dec. 2013.

[9] G. Fornaro, D. Reale, and S. Verde, "Bridge thermal dilation monitoring with millimeter sensitivity via multidimensional SAR imaging," IEEE Geosci. Remote Sens. Lett., vol. 10, no. 4, pp. 677-681, Jul. 2013.

[10] O. Monserrat, M. Crosetto, M. Cuevas, and B. Crippa, "The thermal expansion component of persistent scatterer interferometry observations," IEEE Geosci. Remote Sens. Lett., vol. 8, no. 5, pp. 864-868, Sep. 2011.

[11] K. Goel, F. R. Gonzalez, N. Adam, J. Duro, and M. Gaset, "Thermal dilation monitoring of complex urban infrastructure using high resolution SAR data," in Proc. IEEE Geosci. Remote Sens. Symp., Quebec, Canada, 2014, pp. 954-957.

[12] M. Cuevas, O. Monserrat, M. Crosetto, and B. Crippa, "A new product from persistent scatterer interferometry: The thermal dilation maps," in Proc. Joint Urban Remote Sens. Event, 2011, pp. 285-288.

[13] D. Perissin and F. Rocca, "High-accuracy urban DEM using permanent scatterers," IEEE Trans. Geosci. Remote Sens., vol. 44, no. 11, pp. 3338-3347, Nov. 2006.

[14] M. Crosetto, O. Monserrat, R. Iglesias, and B. Crippa, "Persistent scatterer interferometry: Potential, limits and initial C- and X-band comparison," Photogramm. Eng. Remote Sens., vol. 76, no. 9, pp. 1061-1069, Sep. 2010.

[15] S. Gernhardt, N. Adam, M. Eineder, and R. Bamler, "Potential of very high resolution SAR for persistent scatterer interferometry in urban areas," Ann. GIS, vol. 16, no. 2, pp. 103-111, Jun. 2010.

[16] R. Bamler, M. Eineder, A. Nico, X. Zhu, and S. Gernhardt, "Interferometric potential of high resolution spaceborne SAR," Photogrammetrie - Fernerkundung - Geoinf, vol. 2009, no. 5, pp. 407-19, 2009.

[17] T. Wang, D. Perissin, M. Liao, and F. Rocca, "Three gorges dam stability monitoring with time-series InSAR image analysis," Sci. China Earth Sci., vol. 54, no. 5, pp. 720-732, 2010.

[18] J. J. Sousa, M. Lazecky, I. Hlavacova, M. Bakon, G. Patricio, and D. Perissin, "Satellite SAR interferometry for monitoring dam deformations in Portugal," presented at the Dam World, Lisbon, Portugal, Apr. 2015.

[19] S. Gernhardt and R. Bamler, "Structural deformation and non-seasonal motion of single buildings in urban areas revealed by PSI," presented at the Joint Urban Remote Sensing Event, Lausanne, Switzerland, Apr. 2015.

[20] S. S. Esfahany, R. Hanssen, K. van Thienen-Visser, and A. MuntendamBos, "On the effect of horizontal deformation on InSAR subsidence estimates," presented at the Fringe, Frascati, Italy, 2009.

[21] I. Cumming and J. Zhang, "Measuring the 3-D flow of the Lowell glacier with InSAR," presented at the Fringe, Liége, Belgium, 1999.

[22] C. Colesanti, A. Ferreti, F. Novali, C. Prati, and F. Rocca, "SAR monitoring of progressive and seasonal ground deformation using the permanent scatterers technique," IEEE Trans. Geosci. Remote Sens., vol. 41, no. 1, pp. 1685-1701, Jul. 2003.

[23] M. Lazecky, D. Perissin, M. Bakon, J. J. M. Sousa, I. Hlavacova, and N. Real, "Potential of satellite InSAR techniques for monitoring of bridge deformations," presented at the Joint Urban Remote Sensing Event, Lausanne, Switzerland, Apr. 2015

[24] R. F. Hanssen, Radar Interferometry: Data Interpretation and Error Analysis. Dordrecht, Netherlands: Kluwer, 2001.

[25] I. Hlavacova, J. Kolomaznik, and L. Halounova, "TerraSAR-X staring spotlight monitoring of a highway bridge in the Czech republic," presented at the Fringe, Frascati, Italy, Mar. 2015.

[26] U. Wegmuller and Ch. Werner, "Mitigation of thermal expansion phase in persistent scatterer interferometry in an urban environment," presented at the Joint Urban Remote Sensing Event, Lausanne, Switzerland, Apr. 2015 .

Authors' photographs and biographies not available at the time of publication. 